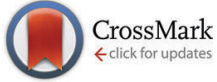

Cite this: Phys. Chem. Chem. Phys., 2015, 17, 25429

Received 24th July 2015, Accepted 2nd September 2015

DOI: $10.1039 / c 5 c p 04353 k$

www.rsc.org/pccp

\title{
100 fs photo-isomerization with vibrational coherences but low quantum yield in Anabaena Sensory Rhodopsin $\dagger$
}

\author{
Alexandre Cheminal, ${ }^{a}$ Jérémie Léonard, ${ }^{\mathrm{a}}$ So-Young Kim, ${ }^{\mathrm{b}}$ Kwang-Hwan Jung, ${ }^{\mathrm{b}}$ \\ Hideki Kandori ${ }^{\complement}$ and Stefan Haacke*a
}

\begin{abstract}
Anabaena Sensory Rhodopsin (ASR) stands out among the microbial retinal proteins in that, under lightadaptation (LA) conditions, it binds both the 13-cis isomer and the all-trans isomer of the protonated Schiff base of retinal (PSBR). In the dark-adapted (DA) state, more than $95 \%$ of the proteins bear all-trans PSBR, and the protein environment adopts a different equilibrium state. We report the excited state and photo-isomerization kinetics of ASR under different LA conditions. The full data set allows confirming that the photoisomerization of the $13 \mathrm{C}$ isomer occurs within $100 \mathrm{fs}$ and indications of an excited and ground state wavepacket launched by the ultrafast non-adiabatic reaction are reported. Even though this recalls the record isomerization time and the coherent reaction scenario of 11-cis PSBR in rhodopsin, the photoisomerization quantum yield (QY) is much lower, actually the lowest value ever reported for retinal proteins $(<15 \%)$. Noticeably, in ASR the excited state lifetime (ESL) is at least five times larger and the QY is more than twice as large for AT PSBR as compared to 13C PSBR. We argue that ESL and QY cannot be expected to be correlated at all, but that the latter is decided on, as often anticipated, by the wavepacket pathways leading to the conical intersection seam.
\end{abstract}

\section{Introduction}

Studying the ultrafast light-induced processes in retinal proteins has attracted vivid attention over the past few years from experimentalists and theoreticians alike. ${ }^{1-7}$ While an initial work revealed the timescales for isomerization of the protonated Schiff base of retinal (PSBR), and how they depend on the protein species or on point mutations, more attention was recently devoted to the mechanism, by which the protein steers this ultrafast reaction. New femtosecond spectroscopic ${ }^{8-19}$ and quantum chemistry tools were devised ${ }^{20-26}$ with the aim of drawing a very detailed picture of the excited state multi-dimensional potential energy surface (PES) including the conical intersection (CI) seam, of the time-resolved motion of PSBR at the atomic level, the reactiondriving vibrational modes and the dynamic changes in the charge distribution on PSBR and in the protein electrostatics. Additional,

\footnotetext{
${ }^{a}$ Institut de Physique et Chimie des Matériaux de Strasbourg \& Labex NIE, Université de Strasbourg - CNRS, 67034 Strasbourg, France. E-mail: haacke@unistra.fr

${ }^{b}$ Department of Life Science and Institute of Biological Interfaces, Sogang University, Shinsu-Dong 1, Mapo-Gu, Seoul 121-742, South Korea ${ }^{c}$ Department of Frontier Materials, Nagoya Institute of Technology, Showa-ku, Nagoya 466-8555, Japan

$\dagger$ Electronic supplementary information (ESI) available. See DOI: 10.1039/ c5cp04353k
}

though partial, insight into these aspects comes from comparative studies of PSBR in solution ${ }^{27-34}$ and of proteins reconstituted with non-isomerizing PSBR derivatives. ${ }^{35-37}$

While the relatively high complexity and molecular interdependencies of the system impair the ability to single out specific molecular or structural factors, there is accumulating evidence that the protein acts to limit the isomerization to one specific double bond of the PSBR backbone and to increase the reaction quantum yield, by steric and electrostatic constraints, preventing ultrafast non-productive routes ${ }^{38}$ or possibly modifying the conical intersection (CI) topology. ${ }^{22,39,40}$

Nevertheless, the process by which the protein environment tunes the excited state PES or CI so as to shorten the $S_{1}$ lifetime and accelerates the reaction remains an open question. Some hints come from the comparison of rhodopsin (rho) with microbial retinal proteins (MRPs), among which bacteriorhodopsin (bR) is by far the most intensively studied representative. Scheme 1 summarizes the photo-reaction scheme of all-trans, 15-anti PSBR in light-adapted bR and in other MRPs, where the PSBR is accommodated almost exclusively in an all-trans form undergoing $100 \%$ bond-specific isomerization around the methyl-substituted $\mathrm{MeC13}=\mathrm{C} 14 \mathrm{H}$ double-bond, whereas the 11-cis PSBR of rhodopsin isomerizes exclusively around the non-substituted $\mathrm{HC11}=\mathrm{C} 12 \mathrm{H}$ double bond. The average excited state lifetimes (ESL) are sub-ps and display single or bi-exponential character depending on the RP 


$$
P S B R_{A T / 15 A}^{*}(F C) \underset{<50 f s}{\rightarrow} I_{A T / 15 A} \underset{E S L}{\rightarrow} C I \rightarrow\left\{\begin{array}{c}
\stackrel{\eta_{A T}}{\rightarrow} J_{13 C / 15 A} \underset{3-5 p s}{\rightarrow} K_{13 C / 15 A} \rightarrow \\
\underset{1-\eta_{A T}}{\rightarrow} P S B R_{A T / 15 A}
\end{array} \rightarrow\right.
$$

Scheme 1 Reaction sequence for AT-to-13C photoisomerization in light-adapted MRPs other than ASR. Photoexcitation creates an excited state population PSBR* in the Franck-Condon region. Bond-length alternation, charge translocation and subsequent vibrational relaxation populates a transient intermediate (the I intermediate) that decays, with an excited state lifetime (ESL) and after passage of the conical intersection (CI) into the product isomer (states $\mathrm{J} \& \mathrm{~K}$ ) or into the reactant isomer, according to the reaction quantum yields $\eta_{\text {AT }}$ and $\eta_{13 \mathrm{C}}$.

studied. In MRPs and rho the photoisomerization yields are similarly high $(\sim 2 / 3)$, but the ESL of rhodopsin $(<0.15 \mathrm{ps})$ is shorter than that of other MRPs and bR in particular $(\sim 0.5 \mathrm{ps})$.

The ground state pretwist of the isomerizing bond ${ }^{41}$ has been suggested to explain the very fast photoreaction of rho. ${ }^{42,43}$ Enhanced Raman activity in the hydrogen-out-of plane (HOOP, $\mathrm{C} 11-\mathrm{H}$ and $\mathrm{C} 12-\mathrm{H}$ ) vibrational modes in $\mathrm{rho}^{44-46}$ was interpreted as a signature of the predominance of these modes in the fast reactive motion towards the $\mathrm{CI},{ }^{11}$ possibly controlling the branching between reactive and abortive decay pathways. ${ }^{11,25}$ Another singularity of rhodopsin's photoreactivity are the characteristic spectroscopic features unraveling a pronounced, coherent, wavepacket-type vibrational motion on the $\mathrm{S}_{1}$ and $\mathrm{S}_{0}$ PESs. ${ }^{18,47}$

In the case of bR, the X-ray crystallographic data of highest resolution also report a significant pretwist of the isomerizing $\mathrm{C} 13=\mathrm{C} 14$ bond, ${ }^{48,49}$ although other published structures or results do not confirm it. ${ }^{50,51}$ Certainly in bR, no HOOP Raman signatures similar to that of rho but rather the methyl rocking mode of Me-C13 are observed in the ground state, ${ }^{52}$ and no vibrationally-coherent reaction mechanism, i.e. vibrational coherence surviving in the ground state after passage of the $\mathrm{CI}$, was clearly reported in MRPs, apart from bR when probed in the near-UV. ${ }^{10}$

Common to all protonated Schiff bases and retinal proteins, the fundamental $\pi \rightarrow \pi^{*}$ bond length alternation (BLA) occurs together with a photo-induced charge translocation in $\mathrm{S}_{1}{ }^{53}$ and unlocks torsional motion around the isomerizing bond. BLA is thus a major contribution of the early vibrational motion out of the Franck-Condon region. However, in bR further evolution towards the CI occurs along a flatter $\mathrm{S}_{1}$ PES including a tiny energy barrier likely originating from the proximity of and mixing with the $S_{2}$ state (the "three state model"), ${ }^{49,54}$ while the $\mathrm{S}_{1}$ character of PSBR in rho would be closer to that of PSBR in a vacuum, ${ }^{21}$ i.e. well accounted for by a two-state model yielding a barrier-less $\mathrm{S}_{1}$ PES. $^{55}$

The recently discovered Anabaena Sensory Rhodopsin (ASR) offers a very interesting test ground in that context. ${ }^{56-60}$ It bears a mixture of all-trans, 15-anti (AT) and 13-cis, 15-syn (13C) PSBR isomers, the relative content of which depends on the illumination, so-called 'light-adaptation' conditions. When left in the dark, the dark-adapted (DA) form adopts $97 \%$ AT, which drops to less than $40 \%$ under illumination with orange light, at neutral $\mathrm{pH}^{57,61}$

Recent ultrafast transient absorption (TA) experiments ${ }^{59}$ indicate that the photoreactions of AT and 13C differ largely in their excited state lifetimes and subsequent photo-product formation. While the AT form displays bR-like kinetics with a double exponential decay $\left(0.5\right.$ and $\left.1.5 \mathrm{ps}^{62}\right)$, the excited state decay of $13 \mathrm{C}$ shows a strong sub-150 fs time component. ${ }^{59}$ Thus, $13 \mathrm{C}$ has an excited state lifetime (ESL) similar to the hitherto fastest known photo-isomerization reaction namely 11-cis PSBR in rhodopsin. These findings were in qualitative agreement with quantum chemistry computations of the excited state PES along the minimum energy paths in $\mathrm{ASR}^{58}{ }^{58}$ and with excited state trajectory calculations. ${ }^{60}$ There it was found that the $\mathrm{S}_{1}$ PES of 13C is barrierless, while that of the AT form displays a plateau or a slight barrier that could account for a short resting period of the excited state population. Importantly, both reaction paths lead to a conical intersection seam with the ground state PES. The precise origin of these different shapes of the $\mathrm{S}_{1}$ PES for both isomers remains, however, unclear.

Here, we report on ultra-broadband probed TA experiments performed with $<60 \mathrm{fs}$ time resolution. The TA spectra and kinetics obtained under excitation at $560 \mathrm{~nm}$ qualitatively confirm the results reported by Ruhman and collaborators. ${ }^{59}$ We present in addition a careful inspection of the time-resolved differential spectra and kinetics. This offers an alternative to studying isomer-dependent evolution associated difference spectra. Those have the advantage of reducing the complexity of the 2D data set, but rely on strong assumptions like the validity of global fitting. When the 100 fs ESL $13 \mathrm{C}$ form is excited, small amplitude vibrational wavepackets are observed, most likely in the photoproduct ground state with a $\approx 0.5-0.6 \mathrm{ps}$ period, very similar to the one reported for rho, ${ }^{47}$ though less pronounced. They are the natural extension of the excited state wavepacket observed by dynamic spectral shifts of the excited state absorption (ESA) and stimulated emission (SE) bands. 13C in wt-ASR thus seems to be another example of a vibrationally coherent isomerization reaction. ${ }^{18,47,63}$

Besides, a precise analysis of the final (ns) TA spectra in terms of equilibrated isomer difference spectra reveals an unexpectedly low reaction quantum yield (QY) of $<0.3$ for both the AT and $13 \mathrm{C}$ isomers. This result, again hinting at the lack of correlation between the ESL and QY, is in line with similar reports on PSBR in solution. ${ }^{19,34,39}$ or on rhodopsin-mimicking photo-switches, ${ }^{64}$ and calls for explanation. Indeed, these findings are at odds with previous reports ${ }^{43}$ on different retinal forms in rhodopsin concluding that a reduction in ESL would imply an increase in $\mathrm{QY}$, as a general consequence of the Landau-Zener formula for non-adiabatic internal conversion. ${ }^{65}$

\section{Experimental methods}

ASR was prepared according to reported protocols ${ }^{24,31}$ and solubilized in buffer ( $200 \mathrm{mM} \mathrm{NaCl}, 25 \mathrm{mM}$ Tris-HCL, $0.05 \%$ DDM, $\mathrm{pH}$ 7.0). The isomer content of the sample at $\mathrm{pH} 7.0$ was determined by HPLC analysis, performed as previously described, ${ }^{57}$ but with a few modifications. Extraction of retinal oxime from the sample was carried out with hexane after adding hydroxylamine (at least 1000-fold molar excess with 
Table 1 Results of HPLC analysis: the PSBR isomer content (percentages) under different light-adaptation conditions (average of 3 runs for $O A$ and BA samples and 4 runs for the DA sample)

\begin{tabular}{lll}
\hline & All-trans, 15-anti $(\mathrm{AT})$ & 13-cis, 15-syn $(13 \mathrm{C})$ \\
\hline DA & $95.7( \pm 0.5)$ & $4.3( \pm 0.5)$ \\
OA & $35.5( \pm 1)$ & $64.5( \pm 1)$ \\
BA & $45.3( \pm 0.3)$ & $54.7( \pm 0.3)$
\end{tabular}

respect to the pigment) and denaturation with ethanol. This procedure was carried out in red light, either directly for darkadapted (DA) samples or after light adaptation under illumination with an orange $(590 \mathrm{~nm})$ or a blue $(490 \mathrm{~nm}) \mathrm{LED}$, for orange-adapted (OA) and blue-adapted (BA) samples respectively. The samples were analyzed on a Purospher STAR Si-5 $\mu \mathrm{m}$ (LichroCART 250-4, Merck) analytical column on a Waters 1525 HPLC equipped with a Waters 2487 Dual $\lambda$ absorbance detector. The solvent was composed of $12(\mathrm{v} / \mathrm{v}) \%$ ethyl acetate and $0.12(\mathrm{v} / \mathrm{v}) \%$ ethanol in hexane, and the flow rate was $1.0 \mathrm{~mL} \mathrm{~min}^{-1}$. The molar compositions of the retinal isomers were calculated from integration over the peak areas in the HPLC patterns. Retinal oxime isomers exist in 15-syn and 15-anti forms; therefore, there are two isomers for all-trans (with retention times of 6.1 and $12.8 \mathrm{~min}$ ) and two for 13-cis (6.9 and $8.0 \mathrm{~min})$. The sum of integrated areas of both 15-syn and 15-anti was used for isomeric ratio calculations. The results of HPLC shown in Table 1 are obtained by averaging at least three independent experiments for the three different light adaptations used. The results are in good agreement with ref. 59 and 61

The pump probe setup for transient absorption (TA) detection has been described in detail elsewhere.$^{63}$ Briefly, it uses the $40 \mathrm{fs}$ pulses of a Ti:sapphire regenerative amplifier ( $5 \mathrm{kHz}$ ) to pump a TOPAS providing approximately $40 \mathrm{fs}$ long pulses at $560 \mathrm{~nm}$. The probe white-light continuum is generated in a $2 \mathrm{~mm} \mathrm{CaF}_{2}$ plate. Pump and probe beams were co-linearly polarized. The solubilized ASR was circulated through a $0.2 \mathrm{~mm}$ path length fused silica flow cell using a peristaltic pump. The sample absorbance at the absorption maximum was in the range of 4 to $8 \mathrm{~cm}^{-1}$ and the excitation density was about $10 \%$ (below $0.5 \mathrm{~mJ} \mathrm{~cm}^{-2}$ per pulse, i.e. in the linear excitation regime). Orange or blue light adaptation was obtained by continuous illumination of the transparent container, with the same LEDs as used for HPLC analysis (see above), with high enough intensities so as to stabilize the isomer content of ASR. Dark adaptation was obtained by leaving the sample in the dark over more than $12 \mathrm{~h}$ at room temperature (the time constant for dark adaptation is about $3 \mathrm{~h}^{66}$ ) and using a light-sealed container during TA experiments.

Separate runs of experiment with the solvent alone were used to subtract signal contributions due to the solvent or flow cell and to correct for the white-light group velocity dispersion $\left(20 \mathrm{fs}\right.$ accuracy). ${ }^{63}$ The time resolution of the experiment defined by the FWHM of the solvent Raman response was determined to be 50 to 60 fs. The ASR solutions used in the present pump-probe experiments displayed a relatively high scattering background. Therefore, a $30 \mathrm{~nm}$ broad region centered on the excitation wavelength is still dominated by pump beam scattering, and has to be partly disregarded. All decay traces are fitted by multi-exponential functions convolved with the IRF approximated as a Gaussian. ${ }^{63}$

\section{Results}

\section{Analysis of ground state absorption spectra}

From an ASR solution with fixed molar concentration, we retrieve the molar absorption spectra $A_{13 \mathrm{C}}$ and $A_{\mathrm{AT}}$ of pure $13 \mathrm{C}$ and AT isomers of ASR from the steady-state absorption spectra of the DA and OA ASR solutions based on the precise determination of their isomer content (see Table 1), and according to:

$$
\left[\begin{array}{c}
A_{\mathrm{AT}} \\
A_{13 \mathrm{C}}
\end{array}\right]=R^{-1} \times\left[\begin{array}{c}
A_{\mathrm{DA}} \\
A_{\mathrm{OA}}
\end{array}\right] \text { with } R=\left[\begin{array}{cc}
r_{\mathrm{DA}}^{\mathrm{AT}} & r_{\mathrm{DA}}^{13 \mathrm{C}} \\
r_{\mathrm{OA}}^{\mathrm{AT}} & r_{\mathrm{OA}}^{13 \mathrm{C}}
\end{array}\right]
$$

$R$ is a $2 \times 2$ matrix given by the first two lines of Table 1 . The isomer specific absorption spectra of pure $13 \mathrm{C}$ and AT ASR are calculated according to eqn (1) and scaled against the value at the isobestic point at $485 \mathrm{~nm} .{ }^{66}$ Taking into account the molar concentration, Fig. 1 shows the so-determined molar extinction coefficients. The excellent agreement between the reconstructed and measured BA spectra provides an indication of the accuracy of the method (see Fig. S1, ESI $\dagger$ ).

On the basis of these results, the relative content of excited isomers as a function of excitation wavelength $\lambda$ can be calculated for different light-adaptation conditions, taking into account the spectral lineshape $P(\lambda)$ of the $560 \mathrm{~nm}$ pump pulses (shaded in Fig. 1). As an example, for the AT content under the OA conditions the formula is

$$
\begin{aligned}
& r_{\mathrm{AT}}{ }^{*}(\mathrm{OA})=\frac{r_{\mathrm{AT}}^{\mathrm{OA}} \int P(\lambda) \varepsilon_{\mathrm{AT}}(\lambda) \mathrm{d} \lambda}{\int P(\lambda)\left[r_{13 \mathrm{C}}^{\mathrm{OA}} \varepsilon_{13 \mathrm{C}}(\lambda)+r_{\mathrm{AT}}^{\mathrm{OA}} \varepsilon_{\mathrm{AT}}(\lambda)\right] \mathrm{d} \lambda} \\
& r_{13 \mathrm{C}^{*}}(\mathrm{OA})=1-r_{\mathrm{AT}}{ }^{*}(\mathrm{OA})
\end{aligned}
$$

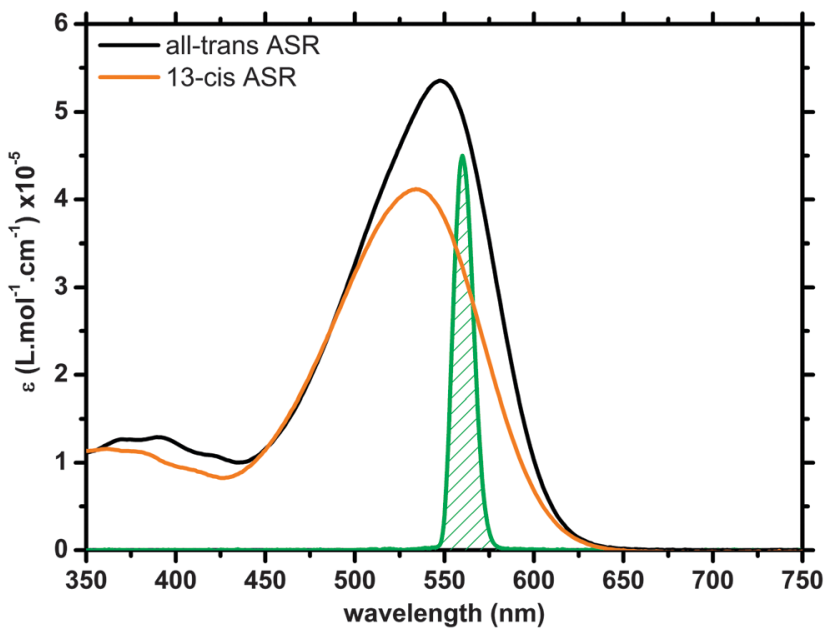

Fig. 1 Extinction coefficients of ASR in both isomeric forms, computed from the spectra of dark- and orange-adapted ASR and their isomer composition obtained by HPLC. The green filled curve represents the $560 \mathrm{~nm}$ excitation pulse spectrum 
We will use this isomeric fraction in the excited state to separate isomer specific contributions from the transient absorption data (see below).

\section{Femtosecond transient absorption}

\section{Time-resolved spectra}

a. Effect of light-adaptation on the excited state and photoproduct differential spectra. Fig. 2 displays the UV-Vis (370$900 \mathrm{~nm}$ ) TA spectra of DA (left, panels a $+\mathrm{c}$ ) and OA (right) ASR, excited at $560 \mathrm{~nm}$ for a selection of delay times shorter than 5 ps (the full two-dimensional data sets are plotted in Fig. S2, ESI $\dagger$ ). Light-adaptation has essentially no effect on the TA spectra: Both DA and OA data are dominated by an intense excited state absorption (ESA, max. at $475 \mathrm{~nm}$ after an initial blue-shifting), ground state bleach (GSB, max. at 550-560 nm), and a broad stimulated emission (SE) band from 650 until $900 \mathrm{~nm}$ and beyond. A low-energy ESA overlaps the broad SE resulting in the positive peak at around $770 \mathrm{~nm}$. A similar low-energy ESA signature was already reported for bR. ${ }^{54,63}$

During the first 50-100 fs the SE red-shifts and stabilizes with a maximum at $850 \mathrm{~nm}$ and the dominant blue ESA blue-shifts, in analogy with reported spectral signatures of the excited state dynamics of $\mathrm{bR},{ }^{54,62,67,68}$ and assigned to excited state relaxation following excited state bond length alternation.
A photo-induced absorption rises on the 100 fs to 200 fs timescale at around $620 \mathrm{~nm}$ and competes with the vanishing SE, which red-shifts and rises in the red-most part of the detection spectral window. Since this time range has all (DA) or most (OA) proteins in the excited state, this should be attributed to an early ESA. Then, as the SE and ESA contributions decay, the early $620 \mathrm{~nm}$-ESA presumably also decays and is replaced by the photoproduct absorption (usually called "J") that emerges fully at $620-630 \mathrm{~nm}$ for $t>200 \mathrm{fs}$. The latter further narrows and blue-shifts, due to vibrational relaxation on a 3-5 ps timescale (the formation of the relaxed " $K$ " state). Note that, while for the DA sample, $\mathrm{K}$ is almost $100 \% 13$-cis, 15-anti, the latter referred to as $\mathrm{K}_{\mathrm{AT}}$, it is a mixture of 13-cis, 15-anti $\left(\mathrm{K}_{\mathrm{AT}}\right)$ and all-trans, 15-syn $\left(\mathrm{K}_{13 \mathrm{C}}\right)$ isomers in the $\mathrm{OA}$ proteins. If not specified, $\mathrm{K}$ will refer in the following to a LA-dependent isomer composition of the photoproduct. After approximately 5 ps and until at least $5 \mathrm{~ns}$ (the largest time delay achievable in our set-up), the TA signal is stationary (data not shown) and composed of the positive $\mathrm{K}$ species absorption and the negative contribution of GSB (Fig. 3).

While light-adaptation has little effect on the ESA, SE and GSB spectra, it induces however marked effects on their kinetics. Since the 13C isomer undergoes a much faster reaction than $\mathrm{AT},{ }^{59,62}$ the OA sample exhibits an accelerated decay of the SE and
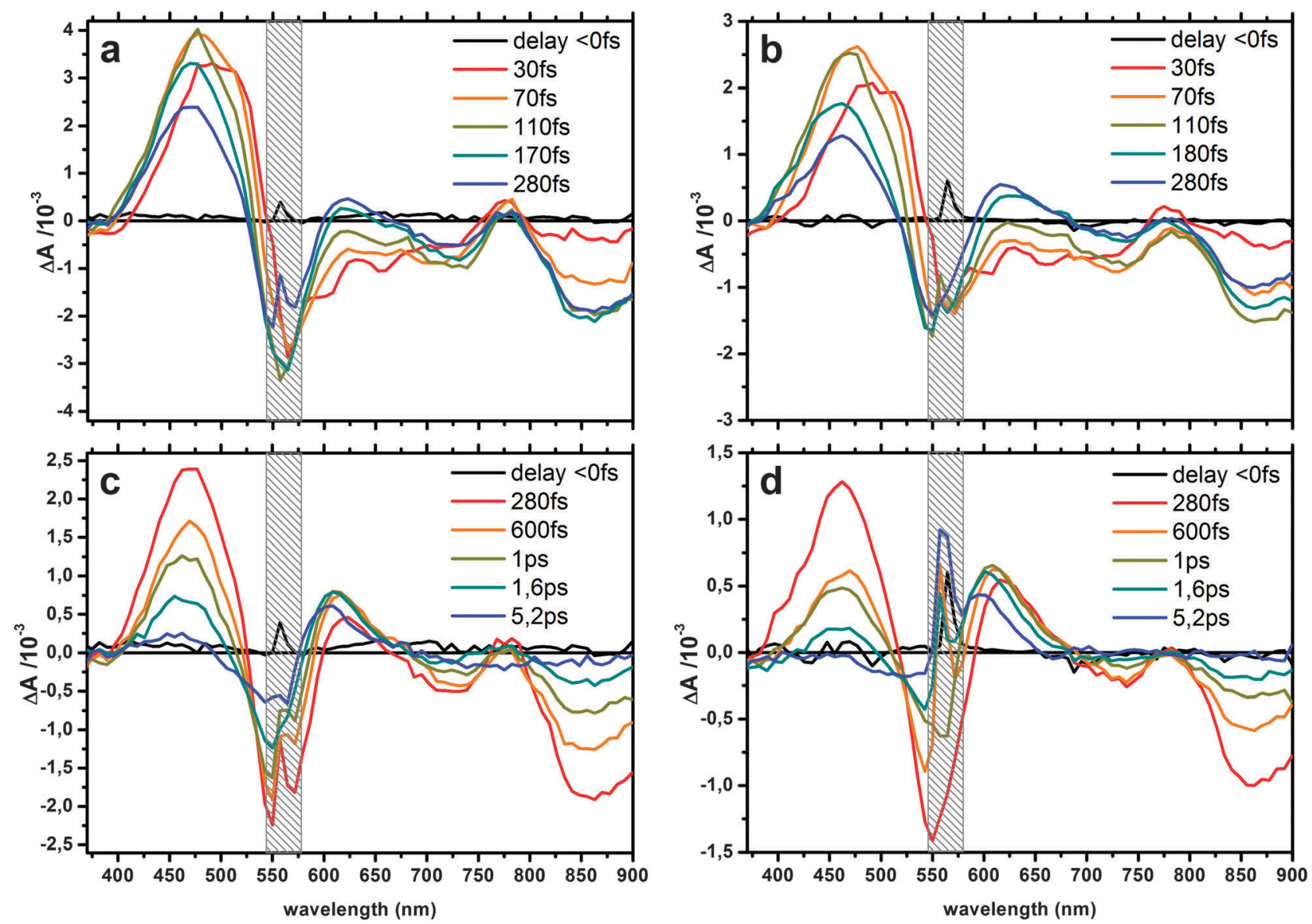

Fig. 2 Transient spectra at early ( $a$ and b) and intermediate ( $c$ and d) times for $560 \mathrm{~nm}$ excitation of DA ASR (a and c), and corresponding spectra of OA ASR ( $b$ and $d)$. The $560 \mathrm{~nm}$ region is disregarded due to the low signal-to-noise ratio by pump scattering. 


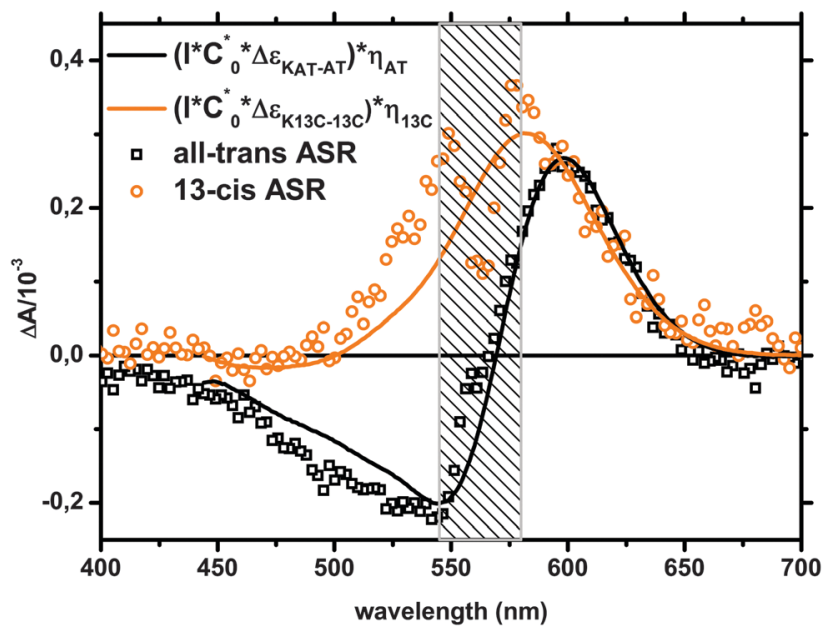

Fig. 3 Long time ( $t>100 \mathrm{ps}$ ) difference spectra of both retinal isomers for $560 \mathrm{~nm}$ excitation obtained after the extraction of pure isomer contribution using eqn (1) and (2). Open symbols represent the TA data and lines are fits using the $\mathrm{K}-\mathrm{GS}$ differential molar extinction coefficients $(\Delta \varepsilon)$ of the respective retinal isomers. The model curves are adjusted using the isomerization quantum yield as a parameter in eqn (3). The present curves yield $\eta_{\mathrm{AT}}=0.13$ and $\eta_{13 \mathrm{C}}=0.05$.

the blue ESA, and a faster rise of the induced absorption in the 620-650 nm range (Fig. 2c). In contrast, the sub-100 fs kinetics are rather independent of light-adaptation conditions, with the simultaneous blue ESA narrowing and blue-shifting and simultaneous SE red shifting. Once spectrally stabilized, both display a fast ( $\approx 50 \%$ intensity) drop in 280 fs for both DA and OA. These differences in the kinetics of ESA and SE will be analyzed in detail below.

b. Long-delay difference spectra and determination of the reaction quantum yield. As the difference spectra are stationary for delays $t \geq 10 \mathrm{ps}$, we average the data in order to obtain the K-ground state (GS) difference spectra for both DA and OA. We show in the ESI $\dagger$ (Fig. S7) that these spectra are identical for different pump intensities and different strains of protein (Kandori vs. Jung lab), but they depend on the light-adaptation conditions, as already reported at $130 \mathrm{~K}^{57}$ and at room temperatures. ${ }^{59}$ However, using eqn (1) and (2), we, can recover the isomer-specific, stationary difference spectra $\Delta A_{\mathrm{AT}}$ and $\Delta A_{13 \mathrm{C}}$ displayed in Fig. 3 , from those of the OA and DA ASR samples investigated under the same experimental conditions (i.e. same pump energy $E_{\mathrm{p}}$ and excitation volume). Provided the average concentration of proteins excited per pulse $C_{0}{ }^{*}$ and the differential extinction coefficients $\Delta \varepsilon_{\mathrm{K}-\mathrm{GS}}^{13 \mathrm{C}}=\varepsilon_{\mathrm{K}}^{13 \mathrm{C}}-\varepsilon_{\mathrm{GS}}^{13 \mathrm{C}}$ and $\Delta \varepsilon_{\mathrm{K}-\mathrm{GS}}^{\mathrm{AT}}=\varepsilon_{\mathrm{K}}^{\mathrm{AT}}-\varepsilon_{\mathrm{GS}}^{\mathrm{AT}}$ are known, one can then infer the isomer-specific reaction $\mathrm{QYs} \eta_{13 \mathrm{C}}$ and $\eta_{\mathrm{AT}}$ according to

$$
\Delta A_{\mathrm{AT}}=C_{0}{ }^{*} l \Delta \varepsilon_{\mathrm{K}-\mathrm{GS}}^{\mathrm{AT}} \eta_{\mathrm{AT}} \text { and } \Delta A_{13 \mathrm{C}}=C_{0}{ }^{*} l \Delta \varepsilon_{\mathrm{K}-\mathrm{GS}}^{13 \mathrm{C}} \eta_{13 \mathrm{C}}
$$

Here, $l$ is the $0.2 \mathrm{~mm}$ path length of the flow cell and the concentration of excited species $C_{0}{ }^{*}$ is given by the number of absorbed photons in a one-photon absorption regime, with
$E_{\mathrm{p}}$ the energy per pulse and $V$ the measured excitation volume (see Section SIV, ESI $\dagger$ ):

$$
C_{0}^{*}=\frac{E_{\mathrm{p}}\left(1-10^{-\mathrm{OD}\left(\lambda_{\mathrm{p}}\right)}\right)}{N_{\mathrm{A}} h c V}
$$

Since the extinction coefficients $\varepsilon_{\mathrm{K}}^{13 \mathrm{C}}$ and $\varepsilon_{\mathrm{K}}^{\mathrm{AT}}$ of $\mathrm{K}_{13 \mathrm{C}}$ and $\mathrm{K}_{\mathrm{AT}}$ are unknown, we approximate them from the ones determined at $130 \mathrm{~K} .{ }^{57}$ This procedure consists in broadening and slightly red-shifting of the $130 \mathrm{~K}$ spectra ( $c f$. details given in Section SIV, ESI $\dagger$ ), while assuming the same temperature-induced broadening and shift for the AT and 13-cis ASR spectra. We also assume that the relative amplitude change of the extinction coefficients of GS and $\mathrm{K}$ forms be temperature-invariant. The results are displayed in Fig. 3, together with the experimental data, which are in very good agreement with spectra reported elsewhere. ${ }^{59}$ The highly asymmetric $\Delta \varepsilon_{\mathrm{K}-13 \mathrm{C}}$ difference spectrum, for $13 \mathrm{C}$, with an intense maximum at $585 \mathrm{~nm}$, and a minor negative lobe below $500 \mathrm{~nm}$ is remarkable. It is due to a significantly higher extinction coefficient of the all-trans, 15-syn photoproduct isomer. Good agreement with the experimental shape of the $\Delta \varepsilon$ spectra (Fig. 3) for both isomers validates the extrapolation procedure from low to room temperature, but imperfections remain in the 500-550 $\mathrm{nm}$ range (see below).

Since $C_{0}{ }^{*}$ has a relatively large error bar ( $c f$. Discussion given in the ESI $\dagger$ ), we first adopt a conservative approach and use eqn (3) and $\varepsilon_{\mathrm{K}}^{13 \mathrm{C}}$ and $\varepsilon_{\mathrm{K}}^{\mathrm{AT}}$ extrapolated above to determine with good accuracy the ratio of the isomerization QYs, $R=\frac{\eta_{\mathrm{AT}}}{\eta_{13 \mathrm{C}}}=\frac{\Delta A_{\mathrm{AT}}}{\Delta A_{13 \mathrm{C}}} \times \frac{\Delta \varepsilon_{\mathrm{K}-\mathrm{GS}}^{13 \mathrm{C}}}{\Delta \varepsilon_{\mathrm{K}-\mathrm{GS}}^{\mathrm{AT}}}$. We find a value of $R=2.7 \pm 0.2$. Then the absolute isomerization quantum yields of each retinal isomer in ASR can be assessed by estimating $C_{0}{ }^{*}$ from eqn (4), which requires careful recording of pump beam intensity and cross section. We find $C_{0}{ }^{*}$ values below $10 \mu \mathrm{M}$, i.e. $C_{0}{ }^{*} / C_{0} \leq$ 0.15 , in agreement with the GSB contribution at $t=0$ (Fig. S8, ESI $\dagger$ ), keeping in mind that the latter is partly reduced due to ESA (Fig. 2, panels a and b). Finally, we infer the isomerization QYs: $\eta_{13 \mathrm{C}->\mathrm{AT}}=0.06 \pm 0.03$ and $\eta_{\mathrm{AT}->13 \mathrm{C}}=0.17 \pm 0.08$. These isomerization quantum yields are, to the best of our knowledge, the smallest isomerization quantum yields ever reported for a protein-bound PSBR. The relative error amounts to $\approx 50 \%$ and largely accounts for the uncertainties in the determination of the room temperature spectra of the $\mathrm{K}$ intermediates (upper boundary) and of the values of $C_{0}{ }^{*}$ (lower boundary, see the ESI $\dagger$ ).

Kandori et al. ${ }^{66}$ have measured these values at photoequilibrium with constant illumination for both PSBR forms in ASR. The values found are $0.38 \pm 0.07$ and $0.24 \pm 0.03$ for AT and 13C PSBR, respectively. We find values, which are more than two times lower. Although the estimation of absolute quantum yield with high precision demands a high confidence in the determination of different parameters, our calculations of the absolute isomerization quantum yields rely on careful measurements and should not give rise to important systematic errors. As discussed in the ESI, $\uparrow$ the values calculated for $C_{0}{ }^{*}$ are rather underestimated, meaning that the error bars of the above QY values define strict upper bounds. In addition, the 
value found for the relative isomerization quantum yield, which gets rid of the determination of $C_{0}{ }^{*}$, gives a value of $R=2.7 \pm$ 0.2 , using three different sets of DA and OA ASR samples. The same value obtained from Kandori's absolute quantum yields and the corresponding error bars is $\Phi_{\mathrm{AT}} / \Phi_{13 \mathrm{C}}=1.6 \pm 0.5$, while the Spudich group gives $R \sim 3.3 .^{69}$ The discrepancies may arise from the different experimental methods used for the quantum yield determination. Our method measures the isomerization quantum yield right after photoproduct relaxation, after single excitation of the sample, while other methods rely on a photostationary equilibrium under constant illumination. The latter methods may produce long-lived photoproducts with incomplete protein thermalization, and therefore change the photochemical equilibrium. However Sineshchekov et al. showed that this is not the case in their experiments, possibly explaining the better convergence between their value of $R$ and ours.

II. Photoreaction dynamics. To further understand the dynamics of retinal photo-isomerization in ASR, we focus on the kinetics of ESA, SE, and K formation, which are representative of the key steps of the reaction.

Excited state lifetime and dynamics. The near-infrared region ( $>850 \mathrm{~nm}$ ), where only SE is present is the ideal wavelength for an examination of the excited state lifetime and the effect of light-adaptation on it.

The SE decay curves probed at $875 \mathrm{~nm}$ are displayed in Fig. 4a together with a fit. Despite stochastic fluctuations two lifetimes of $\tau_{1}=100 \pm 10 \mathrm{fs}$ and $\tau_{2}=770 \pm 10 \mathrm{fs}$ are sufficient. The respective amplitudes are given in Table 2 . The DA trace, for which the AT isomers dominate, is mono-exponential in agreement with ref. 59 and 70, well fitted with $\tau_{2}=770 \pm 10 \mathrm{fs}$ only. The OA kinetics, with $54 \%$ relative $13 \mathrm{C}$ concentration in the excited state shows in addition to $\tau_{2}$ a significant (53\%) contribution of $\tau_{1}$. The $13 \mathrm{C}$ isomers thus react within $100 \mathrm{fs}$, i.e. as fast as the 11C PSBR in rhodopsin. However, it cannot be excluded that a small-amplitude longer lifetime similar to $\tau_{2}$ exists also for 13C. Indeed, after isomer decomposition using
Table 2 Parameters for the bi-exponential decay fit of the SE kinetics, probed at $875 \mathrm{~nm}$

\begin{tabular}{lclcl}
\hline & \multicolumn{4}{l}{ Amplitude (\%) } \\
\cline { 2 - 5 } Decay (fs) & DA ASR & OA ASR & AT ASR & 13C ASR \\
\hline $100 \pm 10$ & 0 & 53 & 0 & 89 \\
$770 \pm 10$ & 100 & 47 & 100 & 11 \\
\hline
\end{tabular}

eqn (1) and (2), we find a $11 \%$ amplitude for the $\tau_{2}$ decay in the fit of the $13 \mathrm{C}$ isomer specific kinetic trace (Fig. 4b) that is significantly above the noise level of the experimental data as indicated by the fit residuals. A similar low-amplitude, longerlived fluorescence component was actually observed in rhodopsin as well. ${ }^{71}$

An interesting new observation is reported in Fig. 5, which compares the SE traces with those of ESA recorded at $425 \mathrm{~nm}$. It is obvious that ESA and SE kinetics do not overlay. Even for the "slow" DA proteins, the ESA at $425 \mathrm{~nm}$ shows a $\approx 100$ fs decay time with $40 \%$ amplitude, which indicates an ultrafast signal decay without the presence of 13C. Since this component is absent in the SE, it does not seem to reflect decay of the excited state population. Instead, the ESA signal is seen to blue-shift from at around $500 \mathrm{~nm}$ to the UV range on the 50 to $100 \mathrm{fs}$ timescale as illustrated in Fig. 6a, and the initial decay of the ESA signal appears even faster at $500 \mathrm{~nm}$ and slower at $425 \mathrm{~nm}$ or below (e.g. $400 \mathrm{~nm}$, not shown). Hence we also attribute this very fast, wavelength-dependent initial ESA decay to a signature of its dynamic blue-shift (see also Fig. S2, ESI $\dagger$ ). It is due to the evolution of the wavepacket away from the Franck-Condon region, along the reaction coordinate.

A similar observation (see Fig. 6b) is made for the 13C PSBR where the ESA signal rises later in the UV and lives longer than at $500 \mathrm{~nm}$, although the total ESA lifetime is significantly shorter than in the AT isomer. The blue-shift of the blue-ESA is also highlighted by the dotted lines in the $2 \mathrm{D}$ data plot shown in Fig. S2 (ESI $\dagger$ ). Noticeably, the $875 \mathrm{~nm}$ SE is similarly delayed as illustrated in Fig. 5a and b. The delayed rise of the UV side of
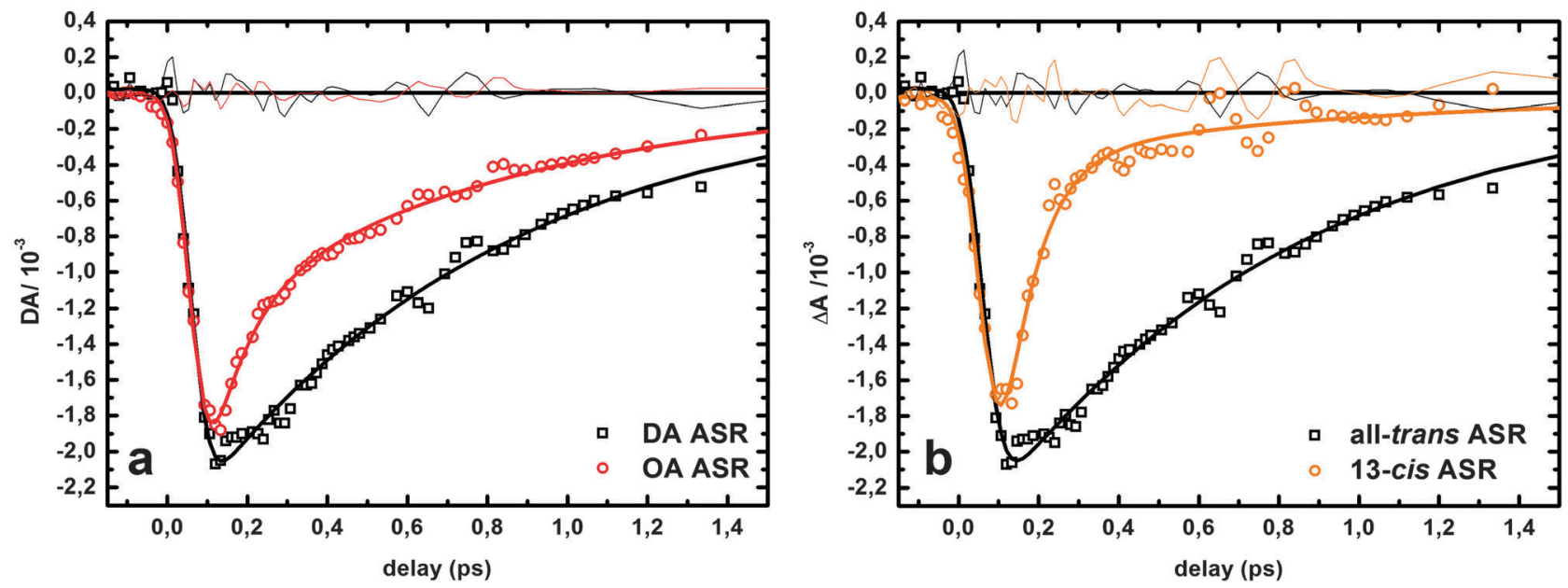

Fig. 4 Transient absorption kinetics of stimulated emission for DA and OA ASR (a) and for AT and 13C PSBR ASR (b) measured at $875 \mathrm{~nm}$. The thick lines are bi-exponential fits ( $\tau_{1}=100 \mathrm{fs}, \tau_{2}=770 \mathrm{fs}$, see Table 2$)$. 

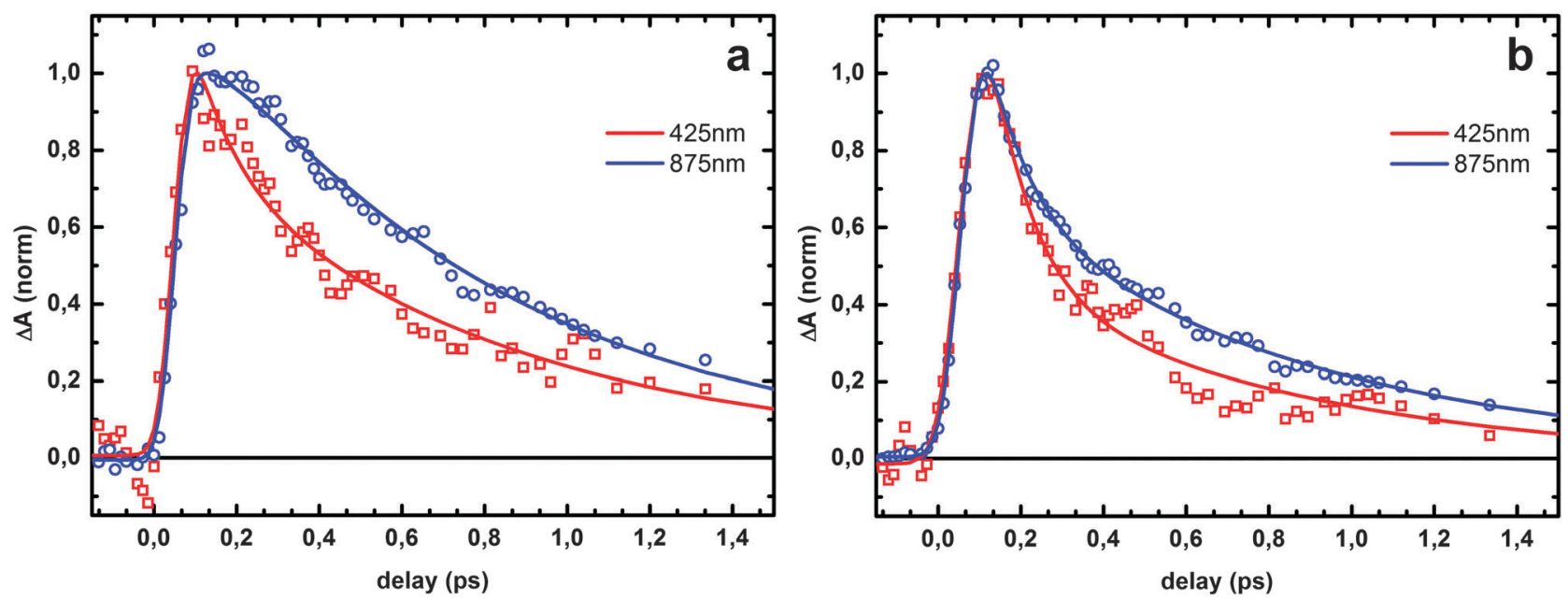

Fig. 5 Transient absorption kinetics of DA (a) and OA (b) ASR excited using a $560 \mathrm{~nm}$ pump. The $425 \mathrm{~nm}$ traces correspond to ESA and the $875 \mathrm{~nm}$ traces probe the SE kinetics, respectively. Thick lines are bi-exponential fits ( $\tau_{1}=100 \mathrm{fs}, \tau_{2}=770 \mathrm{fs}$ ).
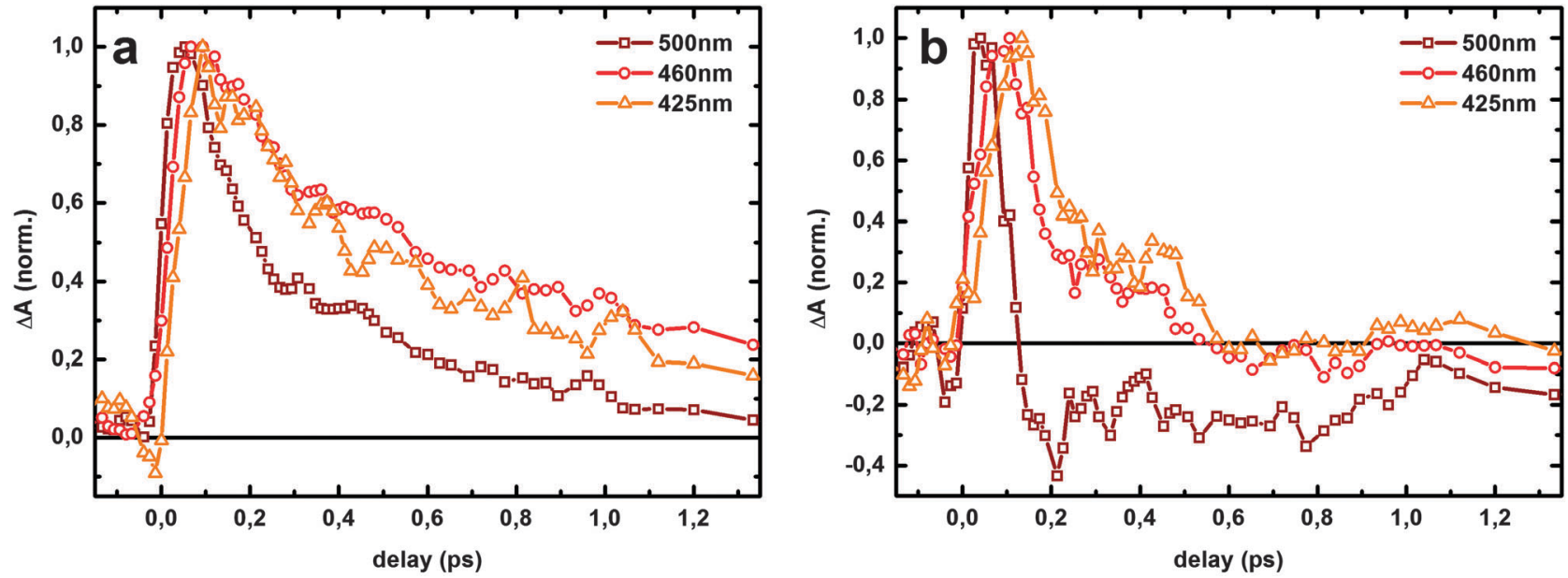

Fig. 6 Normalized transient absorption traces of AT (a) and 13C (b) ASR. The traces represent the ESA and GSB probed at different wavelengths. Note the dynamic spectral shift of the peak wavelength in the first $100 \mathrm{fs}$.

the ESA and of the IR side of the SE is observed both for lightand dark-adapted samples. These observations are common to many retinal proteins and are attributed to the initial relaxation along the $\mathrm{C}=\mathrm{C}$ stretch coordinate as a consequence of bond order change, ${ }^{4-6}$ and possibly along other highfrequency vibrational modes that drive the system away from the Franck-Condon region.

Photoproduct formation. Fig. 7 compares the kinetic traces in the $600-650 \mathrm{~nm}$ range for both the DA and OA samples. They show the cross-over from the SE signal to the formation of the photoproduct J. The SE signal is very short-lived, almost symmetric for both DA and OA since this wavelength region probes the high-energy wing of emission that decays within $\approx 0.1 \mathrm{ps}$ due to the above excited state relaxation. However, the rise of the first photoproduct (J) is slower in DA than for OA, in line with its slower ESL. Global fitting of the 2D data sets determines the relevant timescales of $0.10,0.76$ and $1.6 \mathrm{ps}$ for both
DA and OA, but with amplitudes depending on the lightadaptation conditions, as for ESA and SE. The rise times equal to the excited state lifetimes are determined by the $875 \mathrm{~nm} \mathrm{SE}$ (Fig. 4). The slower 1.6 ps decay times is attributed to vibrational cooling of the ground state species (reactants and the photoproduct), usually referred to as the $\mathrm{J} \rightarrow \mathrm{K}$ transition, and amply documented for retinal proteins in general.

Strikingly, while fitting of the DA transients yields residuals within the signal-to-noise level $\left(\mathrm{rms} 5 \times 10^{-5}\right)$, the $\mathrm{OA}$ residuals (thin lines), and not the DA ones, give evidence for low-frequency oscillations (Fig. 7b). A similar observation is reported in Fig. 8 which displays the residuals of the global fitting of the OA sample at a selection of wavelengths throughout the absorption bands of GS and J. Faint oscillations with a $\approx 0.6-0.7 \mathrm{ps}$ period are observed for the kinetic traces in the $420-650 \mathrm{~nm}$ range superposed on the above double exponential population decay model (cf. Fig. 5b and 7). The first maximum appears at $200 \mathrm{fs}$ on the low-energy side $(>600 \mathrm{~nm})$, together with a minimum in the 

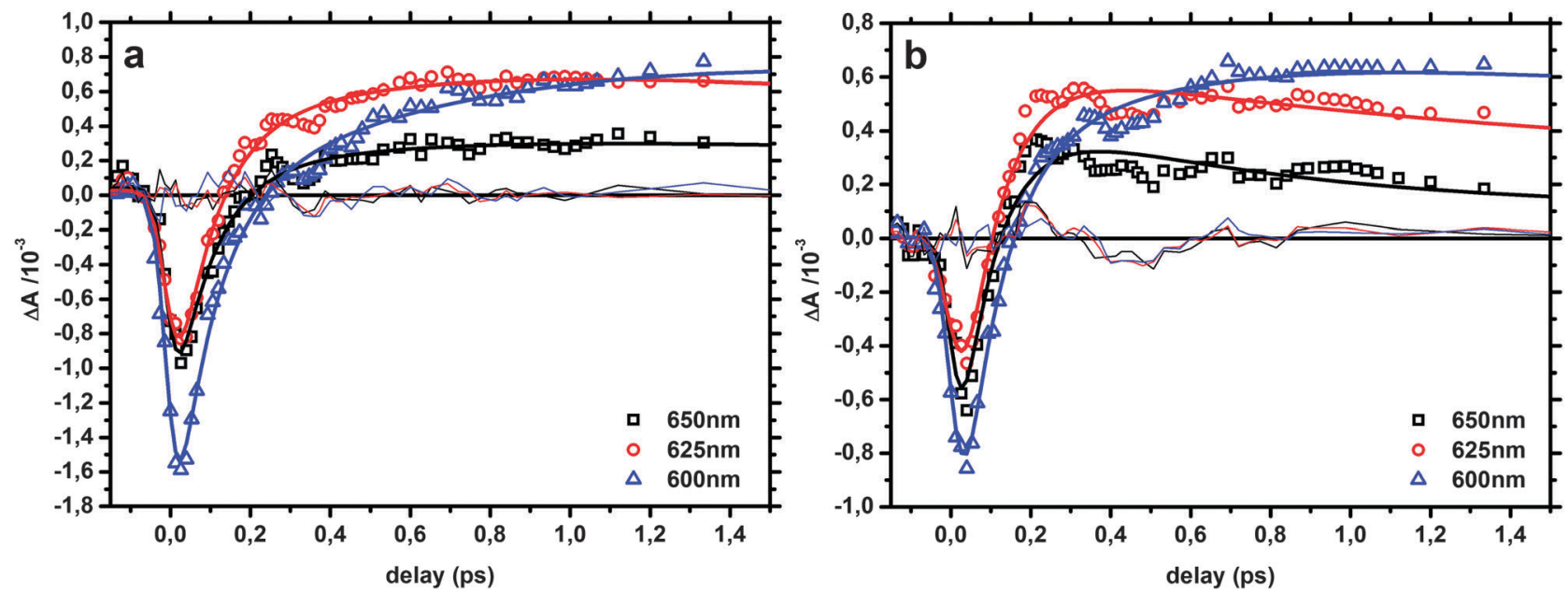

Fig. 7 Transient absorption kinetics of DA (a) and OA (b) ASR for photoproduct dominated spectral regions. Kinetics are fitted with 3-exponential decays, $0.10,0.76$ and $1.6 \mathrm{ps}$ (thick lines). Fit residuals are given as thin lines. Note the appearance of oscillatory features in the fit residuals of OA ASR (b).

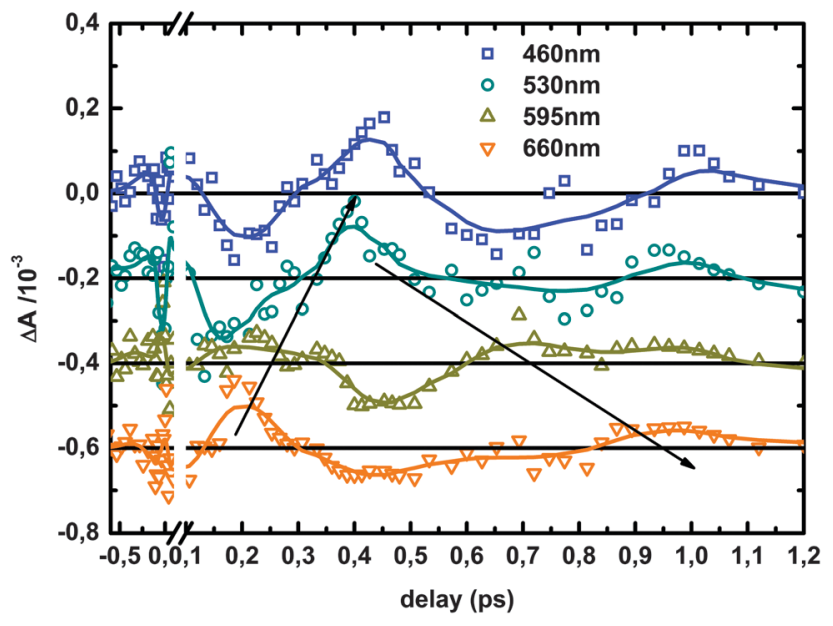

Fig. 8 Oscillatory features found in the residuals of global fits of the OA ASR transient absorption data for different probe wavelengths. These features are significantly higher than the typical noise level at negative delays, and are attributed to ground state wavepackets formed after photo-isomerization. Thick lines are a guide to the eye. Note the opposite sign of phases for the short- and long-wavelength probes.

460-530 nm region. A second appears at 450-500 fs on the high-energy side $(<500 \mathrm{~nm})$, with a concomitant minimum at low energies. It is also clearly observed in the $13 \mathrm{C}$ isomerspecific trace at $425 \mathrm{~nm}$ (Fig. 6b), despite the weaker signal-tonoise ratio resulting from the linear superposition (eqn (1)) that has to be computed to retrieve isomer-specific signals. At longer times, a damped revival is observed (Fig. 8), with a longer-lasting maximum/minimum period, which seems to indicate the coexistence of at least two slightly different vibrational frequencies. Most importantly, the oscillations display opposite phases in the blue and red edges of the associated spectral band, which is characteristic of wavepackets oscillating in the $\mathrm{PES}^{47}$ due to interferences of vibrational states. Within the limited signal-tonoise ratio, one can estimate a damping time in the range of $0.5 \mathrm{ps}$, underscoring the anharmonic character of these oscillations.
The assignment of these oscillatory signals is not trivial. First, since they are not observed in the DA AT-only proteins, excited with the same laser pulses, it seems unlikely that ground state resonance impulsive stimulated Raman scattering (RISRS) $^{72-74}$ is at their origin. Rather they must be related to the reaction of the $13 \mathrm{C}$ isomer. Also these oscillations appear well after the dominant 100 fs excited state deactivation, and their periods are longer than the average 13C ESL. Therefore we tentatively attribute them to a ground state signature (GS or J), that is a ground state wavepacket launched by the $100 \mathrm{fs}$ excited state reactive motion, qualitatively consistent with the ones observed in rho $^{47}$ and the rhodopsin-mimicking NAIP photoswitches. ${ }^{63,75,76}$ However, since the $13 \mathrm{C}$ isomer shows a minor longer-lived excited state decay component (accounting for about $10 \%$ of the population, according to Table 2) we may not completely exclude that the oscillatory signals be a signature of that $10 \%$ fraction of population, which survives longer in the excited state.

\section{Discussion}

The present results can be summarized and discussed as follows.

\section{Isomer-specific reaction kinetics}

In ASR, the photo-isomerization reactions of AT and $13 \mathrm{C}$ give rise to spectrally almost indistinguishable transient absorption signals, except from the final K-GS difference spectra. The excited state lifetimes and reaction rates are however significantly different. While AT decays mono-exponentially with a $0.77 \pm 0.02 \mathrm{ps}$ lifetime, $13 \mathrm{C}$ isomers display a double exponential decay with an average ESL of $170 \pm 40$ fs. These results confirm those recently reported by the Ruhman group, $, 659,62$ in particular, regarding the double component excited state decay behavior for the light-adapted forms and the faster lifetime of the 13C species. The fitted time constants for the ESA are very similar, apart from the 13C related decay time $\left(\tau_{1}\right)$ that appears to be slightly faster in our data, probably due to a different IRF. 


\section{Vibrational wavepackets in $13 \mathrm{C}$ only}

The ultrashort excited state decay of $13 \mathrm{C}$ in ASR is parallel to the one of 11-cis PSBR in rhodopsin and of the 13C isomer in dark-adapted bR. ${ }^{59,67}$ Excited state wavepackets were reported for AT ASR by the Ruhman group. ${ }^{59,62}$ The small amplitude vibrational wavepackets reported in this work for 13C in ASR are most likely in the photoproduct ground states, qualitatively similar to the ones reported in bovine rho. ${ }^{47}$ Such non-exponential signatures are not observed for the AT isomers, whose kinetic traces, due to the longer excited state lifetimes, and within the present signal-to-noise ratio, obey a classical rate equation approach.

If this interpretation is true, these discrepancies between AT and $13 \mathrm{C}$ may be related to the existence of an excited state barrier in the former. ${ }^{58,60}$ The initial 100 fs dynamics of highfrequency modes launches a vibrationally coherent reaction in both isomers, most likely at the origin of the dynamic spectral shifts of ESA and SE observed for both isomers (Fig. 2 and Fig. S2, ESI $\dagger$ ). In 13C, due to the lack of an excited state barrier, the low-frequency wavepacket can fully propagate through the conical intersection and die only in the ground states. As pointed out recently for rhodopsin-mimicking photoswitches, ${ }^{76}$ the existence and survival of vibrational coherences indicates that the reaction coordinate involves only a small set of low-energy vibrational modes with weak coupling to other intra- and intermolecular bath modes.

\section{Low reaction quantum yields}

The reaction quantum yields (QY) of the AT and 13C isomers in ASR are lower than 0.25 . ASR thus stands out among all retinal proteins as one where the reaction yield is not optimized by evolution. This raises interesting fundamental questions as to the mechanical origin, e.g. steric constraints in the protein environment. From quantum chemistry simulations it was inferred that the GS $\rightarrow \mathrm{K}$ photo-isomerization of both $13 \mathrm{C}$ and AT involves a similar counter-clock wise rotation, with respect to the Lys210 side chain, about the $\mathrm{C} 13=\mathrm{C} 14$ bond, ${ }^{58}$ but the QYs or concurrent excited state deactivation paths were not computed.

\section{ESLS and photo-isomerization QYs are not correlated}

Photo-isomerization in retinal proteins is accomplished in less than a picosecond, and as such outperforms any other possible excited state reaction (i.e. intersystem-crossing). The reaction QY is smaller than unity since non-productive internal conversion paths compete with the productive pathways leading to the product isomer ground state.

It was experimentally observed for bovine rhodopsin and its variants, and then extrapolated based on arguments related to the Landau-Zener ( $\mathrm{LZ}$ ) rule for non-adiabatic reactions, that a shorter ESL necessarily entails a higher QY. ${ }^{42,43}$ Our findings are in stark contrast to that: despite a shorter excited state lifetime and indications for partial vibrational coherence, the reaction quantum efficiency is lower in 13C than in $\mathrm{AT}\left(R=\eta_{\mathrm{AT}}\right)$ $\left.\eta_{13 \mathrm{C}}=2.7\right)$. A similar "anti-correlation" between the $\mathrm{S}_{1}$ lifetime and the reaction quantum yield was recently reported for alltrans PSBR and substitutions thereof in solution. ${ }^{34}$

The first reason for this discrepancy with the LZ rule is quite trivial. The latter relates the transition probability with the reaction speed at the transition state. For the non-adiabatic transitions like photo-isomerization in the transition state, namely the conical intersection (CI) is inaccessible by spectroscopic means (vanishing transition dipole moment, sub-10 fs residence time). Worse, the ESL characterizes the reaction speed in the vicinity of the Franck-Condon zone, far away from the CI where the critical transition into the product or reactant ground state PES is decided on. A positive correlation of ES decay rate and QY is therefore only fortuitous.

In addition, and unlike the one-dimensional problem treated by the LZ formalism, concurrent excited and ground state paths in the PES hyperspace have to be accounted for. ${ }^{34,77}$ Recent publications indicate that this problem has to be addressed specifically and separately for different photo-isomerization reactions accounting for the isomer and its particular (protein) environment, through computationally expensive multi-trajectory simulations ${ }^{7,18,34}$

As a case in point, the 9-cis $\rightarrow$ all-trans isomerization in isorhodopsin occurs to be kinetically very similar to the one of $13 \mathrm{C}$ PSBR in ASR. ${ }^{7,78}$ On the basis of hybrid QM/MM trajectories, it was recently proposed that the initial sub-150 fs excited state decay leads to a conical intersection (CI), which turns out to be unproductive for the generation of the all-trans photoproduct. Only the slower $\approx 300 \mathrm{fs}$ small amplitude component leads to a different partly productive CI, and the experimentally observed $22 \%$ QY was quantitatively reproduced by the computed trajectories. ${ }^{78}$ Interestingly, all-trans PSBR in solution also displays a sub-ps ESL component, ${ }^{32}$ which was shown to lead to an unproductive internal conversion (IC) pathway. ${ }^{39}$ These examples illustrate that a precise knowledge of the wavepacket trajectories, and their characterization in terms of transition probabilities to the reactant and product ground states is mandatory in order to quantitatively rationalize the QY values of AT and 13C in ASR.

\section{Conclusion}

Type I and type II RPs are known to enhance the photoisomerization speed and QY of the PSBR isomers they bind with respect to isolated PSBR in solution. ASR is unique among RPs in that it bears both AT and 13C PSBR under light-adaptation conditions, offering thus an interesting model system on how the same protein environment regulates the photochemistry of these two isomers. Here, we show that ASR reduces indeed the excited state lifetimes of both AT and 13C PSBR, with values of, respectively, 0.77 and $0.17 \mathrm{ps}$, in line with values previously reported. ${ }^{59,62}$ The faster excited state deactivation is in line with recent $\mathrm{QM} / \mathrm{MM}$ computations, which identified the existence and absence of an excited state barrier, ${ }^{60}$ most likely induced by a different coupling and mixing of the $S_{1}$ and $S_{2}$ excited states. ${ }^{79}$

Despite these ESL values similar to the ones found in type I and type II RPs, the QYs remain in the range of isolated PSBR, 
namely below $30 \%$. To the best of our knowledge, ASR is the only exception among other RPs in this respect. More specifically, we find that despite a shorter ESL, 13C PSBR in ASR has a lower QY than AT. We provide a series of arguments, which revise the misconception that faster ESLs would entail higher QYs. ESL can by no means be considered as characterizing the speed of the excited wavepacket in the critical regions of conical intersections, where the transition probabilities into the reactant and different photoproduct ground states are decided on. Rather, the present data prompt to the need of a detailed characterization of the excited and ground state wavepacket trajectories and how the PSBR conformation and protein environment affect them. The present and forthcoming experimental results in terms of both QY and vibrational wavepacket properties set a benchmark for such calculations.

\section{Acknowledgements}

The Strasbourg team is grateful to B. Abert and M. Fu for their contribution to the initial phase of the experiments, and to $\mathrm{O}$. Crégut, J.-P. Vola and G. Dekyndt for technical assistance. This work was supported by the ANR grant MolNanoMat (2011-INTB1012-01) and by the Labex NIE.

\section{References}

1 H. J. Polland, M. A. Franz, W. Zinth, W. Kaiser, E. Kölling and D. Oesterhelt, Biophys. J., 1986, 49, 651-662.

2 J. W. Petrich, J. Breton, J. L. Martin and A. Antonetti, Chem. Phys. Lett., 1987, 137, 369-375.

3 J. Herbst, K. Heyne and R. Diller, Science, 2002, 297, 822-825.

4 R. Diller, in Biological and Medical Physics, Biomedical Engineering, ed. M. Braun, P. Gilch and W. Zinth, Springer, Berlin Heidelberg, 2008, pp. 243-273.

5 J. Briand, J. Léonard and S. Haacke, J. Opt., 2010, 12, 084004.

6 A. Wand, I. Gdor, J. Y. Zhu, M. Sheves and S. Ruhman, Annu. Rev. Phys. Chem., 2013, 64, 437-458.

7 D. Polli, I. Rivalta, A. Nenov, O. Weingart, M. Garavelli and G. Cerullo, Photochem. Photobiol. Sci., 2015, 14, 213-228.

8 S. Ruhman, B. X. Hou, N. Friedman, M. Ottolenghi and M. Sheves, J. Am. Chem. Soc., 2002, 124, 8854-8858.

9 G. I. Groma, A. Colonna, J. C. Lambry, J. W. Petrich, G. Váró, M. Joffre, M. H. Vos and J.-L. Martin, Proc. Natl. Acad. Sci. U. S. A., 2004, 101, 7971-7975.

10 S. Schenkl, F. van Mourik, G. van der Zwan, S. Haacke and M. Chergui, Science, 2005, 309, 917-920.

11 P. Kukura, D. W. McCamant, S. Yoon, D. B. Wandschneider and R. A. Mathies, Science, 2005, 310, 1006-1009.

12 D. W. McCamant, P. Kukura and R. A. Mathies, J. Phys. Chem. B, 2005, 109, 10449-10457.

13 J. P. Kraack, T. Buckup, N. Hampp and M. Motzkus, ChemPhysChem, 2011, 12, 1851-1859.

14 J. P. Kraack, T. Buckup and M. Motzkus, Phys. Chem. Chem. Phys., 2012, 14, 13979-13988.
15 V. I. Prokhorenko, A. M. Nagy, S. A. Waschuk, L. S. Brown, R. R. Birge and R. J. D. Miller, Science, 2006, 313, 1257-1261.

16 G. Vogt, P. Nuernberger, T. Brixner and G. Gerber, Chem. Phys. Lett., 2006, 433, 211-215.

17 P. J. M. Johnson, A. Halpin, T. Morizumi, V. I. Prokhorenko, O. P. Ernst and R. J. Dwayne Miller, EPJ Web Conf., 2013, 41, 07020.

18 D. Polli, P. Altoe, O. Weingart, K. M. Spillane, C. Manzoni, D. Brida, G. Tomasello, G. Orlandi, P. Kukura, R. A. Mathies, M. Garavelli and G. Cerullo, Nature, 2010, 467, 440-U488.

19 T. Sovdat, G. Bassolino, M. Liebel, C. Schnedermann, S. P. Fletcher and P. Kukura, J. Am. Chem. Soc., 2012, 134, 8318-8320.

20 L. M. Frutos, T. Andruniow, F. Santoro, N. Ferre and M. Olivucci, Proc. Natl. Acad. Sci. U. S. A., 2007, 104, 7764-7769.

21 T. Andruniów, N. Ferré and M. Olivucci, Proc. Natl. Acad. Sci. U. S. A., 2004, 101, 17908-17913.

22 M. Ben-Nun, F. Molnar, K. Schulten and T. J. Martínez, Proc. Natl. Acad. Sci. U. S. A., 2002, 99, 1769-1773.

23 F. Molnar, M. Ben-Nun, T. J. Martínez and K. Schulten, J. Mol. Struct., 2000, 506, 169-178.

24 A. Cembran, F. Bernardi, M. Olivucci and M. Garavelli, Proc. Natl. Acad. Sci. U. S. A., 2005, 102, 6255-6260.

25 I. Schapiro, M. N. Ryazantsev, L. M. Frutos, N. Ferre, R. Lindh and M. Olivucci, J. Am. Chem. Soc., 2011, 133, 3354-3364.

26 I. Rivalta, A. Nenov, O. Weingart, G. Cerullo, M. Garavelli and S. Mukamel, J. Phys. Chem. B, 2014, 118, 8396-8405.

27 P. Hamm, M. Zurek, T. Röschinger, H. Patzelt, D. Oesterhelt and W. Zinth, Chem. Phys. Lett., 1996, 263, 613-621.

28 S. L. Logunov, M. A. ElSayed, L. Song and J. K. Lanyi, J. Phys. Chem., 1996, 100, 2391-2398.

29 O. Bismuth, N. Friedman, M. Sheves and S. Ruhman, J. Phys. Chem. B, 2007, 111, 2327-2334.

30 O. Bismuth, N. Friedman, M. Sheves and S. Ruhman, Chem. Phys., 2007, 341, 267-275.

31 G. Zgrablić, S. Haacke and M. Chergui, Chem. Phys., 2007, 338, 168.

32 G. Zgrablic, S. Haacke and M. Chergui, J. Phys. Chem. B, 2009, 113, 4384-4393.

33 G. Zgrablic, K. Voitchovsky, M. Kindermann, S. Haacke and M. Chergui, Biophys. J., 2005, 88, 2779-2788.

34 G. Bassolino, T. Sovdat, M. Liebel, C. Schnedermann, B. Odell, T. D. W. Claridge, P. Kukura and S. P. Fletcher, J. Am. Chem. Soc., 2014, 136, 2650-2658.

35 T. Ye, N. Friedman, Y. Gat, G. H. Atkinson, M. Sheves, M. Ottolenghi and S. Ruhman, J. Phys. Chem., 1999, 103, 5122.

36 S. Haacke, S. Schenkl, S. Vinzani and M. Chergui, Biopolymers, 2002, 67, 306-309.

37 S. Haacke, S. Vinzani, S. Schenkl and M. Chergui, ChemPhysChem, 2001, 2, 310-315.

38 G. Zgrablic, S. Haacke and M. Chergui, J. Phys. Chem. B, 2009, 113, 4384-4393.

39 G. Zgrablić, A. M. Novello and F. Parmigiani, J. Am. Chem. Soc., 2011, 134, 955-961.

40 I. Burghardt and J. T. Hynes, J. Phys. Chem. A, 2006, 110, 11411-11423. 
41 T. Okada, M. Sugihara, A.-N. Bondar, M. Elstner, P. Entel and V. Buss, J. Mol. Biol., 2004, 342, 571-583.

42 R. W. Schoenlein, L. A. Peteanu, R. A. Mathies and C. V. Shank, Science, 1991, 254, 412-415.

43 R. W. Schoenlein, L. A. Peteanu, Q. Wang, R. A. Mathies and C. V. Shank, J. Phys. Chem., 1993, 97, 12087-12092.

44 G. Eyring, B. Curry, A. Broek, J. Lugtenburg and R. Mathies, Biochemistry, 1982, 21, 384-393.

45 R. Mathies, A. R. Oseroff and L. Stryer, Proc. Natl. Acad. Sci. U. S. A., 1976, 73, 1-5.

46 J. E. Kim and R. A. Mathies, J. Phys. Chem. A, 2002, 106, 8508-8515.

47 Q. Wang, R. W. Schoenlein, L. A. Peteanu, R. A. Mathies and C. V. Shank, Science, 1994, 266, 422-424.

48 H. Luecke, Science, 1999, 286, 255-261.

49 P. Altoè, A. Cembran, M. Olivucci and M. Garavelli, Proc. Natl. Acad. Sci. U. S. A., 2010, 107, 20172-20177.

50 H. Belrhali, P. Nollert, A. Royant, C. Menzel, J. P. Rosenbusch, E. M. Landau and E. Pebay-Peyroula, Structure, 1999, 7, 909-917.

51 M. F. Brown, M. P. Heyn, C. Job, S. Kim, S. Moltke, K. Nakanishi, A. A. Nevzorov, A. V. Struts, G. F. J. Salgado and I. Wallat, Biochim. Biophys. Acta, Biomembr., 2007, 1768, 2979-3000.

52 M. Braiman and R. Mathies, Biochemistry, 1980, 19, 5421-5428.

53 V. Bonačić-Koutecký, J. Köhler and J. Michl, Chem. Phys. Lett., 1984, 104, 440-443.

54 K. C. Hasson, F. Gai and P. A. Anfinrud, Proc. Natl. Acad. Sci. U. S. A., 1996, 93, 15124-15129.

55 R. González-Luque, M. Garavelli, F. Bernardi, M. Merchán, M. A. Robb and M. Olivucci, Proc. Natl. Acad. Sci. U. S. A., 2000, 97, 9379-9384.

56 L. Vogeley, O. A. Sineshchekov, V. D. Trivedi, J. Sasaki, J. L. Spudich and H. Luecke, Science, 2004, 306, 1390-1393.

57 A. Kawanabe, Y. Furutani, K.-H. Jung and H. Kandori, J. Am. Chem. Soc., 2007, 129, 8644-8649.

58 A. Strambi, B. Durbeej, N. Ferré and M. Olivucci, Proc. Natl. Acad. Sci. U. S. A., 2010, 107, 21322-21326.

59 A. Wand, R. Rozin, T. Eliash, K.-H. Jung, M. Sheves and S. Ruhman, J. Am. Chem. Soc., 2011, 133, 20922-20932.

60 I. Schapiro and S. Ruhman, Biochim. Biophys. Acta, Bioenerg., 2014, 1837, 589-597.

61 R. Rozin, A. Wand, K. H. Jung, S. Ruhman and M. Sheves, J. Phys. Chem. B, 2014, 118, 8995-9006.
62 A. Wand, B. Loevsky, N. Friedman, M. Sheves and S. Ruhman, J. Phys. Chem. B, 2013, 117, 4670-4679.

63 J. Briand, O. Braem, J. Rehault, J. Leonard, A. Cannizzo, M. Chergui, V. Zanirato, M. Olivucci, J. Helbing and S. Haacke, Phys. Chem. Chem. Phys., 2010, 12, 3178-3187.

64 A. Cheminal, J. Leonard, S. Y. Kim, K. H. Jung, H. Kandori and S. Haacke, Chem. Phys. Lett., 2013, 587, 75-80.

65 R. M. Weiss and A. W. Warshel, J. Am. Chem. Soc., 1979, 101, 6131-6133.

66 Y. Wada, A. Kawanabe, Y. Furutani, H. Kandori and H. Ohtani, Chem. Phys. Lett., 2008, 453, 105-108.

67 A. Wand, N. Friedman, M. Sheves and S. Ruhman, J. Phys. Chem. B, 2012, 116, 10444-10452.

68 B. Schmidt, C. Sobotta, B. Heinz, S. Laimgruber, M. Braun and P. Gilch, Biochim. Biophys. Acta, 2005, 1706, 165-173.

69 O. A. Sineshchekov, V. D. Trivedi, J. Sasaki and J. L. Spudich, J. Biol. Chem., 2005, 280, 14663-14668.

70 J. P. Kraack, A. Wand, T. Buckup, M. Motzkus and S. Ruhman, Phys. Chem. Chem. Phys., 2013, 15, 14487-14501.

71 H. Kandori, Y. Furutani, S. Nishimura, Y. Shichida, H. Chosrowjan, Y. Shibata and N. Mataga, Chem. Phys. Lett., 2001, 334, 271-276.

72 U. Banin, A. Bartana, S. Ruhman and R. Kosloff, J. Chem. Phys., 1994, 101, 8461.

73 J. Chesnoy and A. Mokhtari, Phys. Rev. A: At., Mol., Opt. Phys., 1988, 38, 3566.

74 S. L. Dexheimer, Q. Wang, L. A. Peteanu, W. T. Pollard, R. A. Mathies and C. V. Shank, Chem. Phys. Lett., 1992, 188, 61-66.

75 J. Léonard, J. Briand, S. Fusi, V. Zanirato, M. Olivucci and S. Haacke, New J. Phys., 2013, 15, 105022.

76 J. Léonard, I. Schapiro, J. Briand, S. Fusi, R. R. Paccani, M. Olivucci and S. Haacke, Chem. - Eur. J., 2012, 18, 15296-15304.

77 S. Gozem, M. Huntress, I. Schapiro, R. Lindh, A. A. Granovsky, C. Angeli and M. Olivucci, J. Chem. Theory Comput., 2012, 8, 4069-4080.

78 D. Polli, O. Weingart, D. Brida, E. Poli, M. Maiuri, K. M. Spillane, A. Bottoni, P. Kukura, R. A. Mathies, G. Cerullo and M. Garavelli, Angew. Chem., 2014, 53, 2504-2507.

79 H. L. Luk, F. Melaccio, S. Rinaldi, S. Gozem and M. Olivucci, Molecular bases for the selection of the chromophore of animal rhodopsins, 2015, to be published/private communication. 